\title{
Clinico-embryological submission of an unilateral anomalous presentation of axillary artery
}

\author{
Ravi KS, Mehta V, Arora J, Suri RK, Rath G \\ Department of Anatomy, Vardhaman Mahavir Medical College, New Delhi, India. \\ drvandanamehta@gmail.com
}

\begin{abstract}
Objectives: Descriptions of the variant arterial pattern of upper limb are not exceptional and are therefore frequently reported in anatomy archives.

Background: A noteworthy deviation from the usual branching pattern was observed unilaterally in a single cadaver. This unique division of axillary artery (AA) was present on the right side in an adult human cadaver of Indian origin.

Results: The first part of axillary artery gave off the superior thoracic and thoraco-acromial arteries. Just proximal to the upper border of pectoralis minor the AA was observed to divide into two trunks a medial and a lateral. The lateral trunk continued into the brachium as the usual axillary artery where as the medial trunk displayed the other branches deep and distal to the pectoralis minor muscle. The remarkable feature was the wide caliber of the axillary artery where it bifurcated into two branches. An attempt has been made to dwell upon the embryological basis of the present anomaly.

Conclusions: The relevance of anomalous arterial pattern of upper limb (U.L.) is realized while performing percutaneous arterial venous catheter insertion into subclavian vein via the infraclavicular route. We advocate a meticulous familiarization of the anatomy of axillary artery and its topographical relationship to other neurovascular structures for the operating plastic surgeon, anesthetist and radiologist (Fig. 1, Ref. 12). Full Text in PDF www.elis.sk.

Key words: axillary artery, branching, variation, duplication.
\end{abstract}

The classical description of axillary artery (AA) in anatomy text books is that axillary artery is a continuation of subclavian artery at the outer border of 1 st rib and ends by continuing as brachial artery at lower border of teres major (1).

The pectoralis minor muscle divides the AA into three parts and branches of each part are according to the number of the part of axillary artery. So first part has only 1 branch the superior thoracic artery (STA), second part has 2 branches - thoraco-acromial artery (TAA), lateral thoracic artery (LTA) and third part has 3 branches - anterior circumflex humeral artery (AC), posterior circumflex humeral artery (PC) and subscapular artery (SSA).

The present case report highlights a rare duplication of axillary artery in which one trunk (lateral) continues in the brachium while the other trunk (medial) continues itself as the second and third part of the usual AA. We deem this sort of arterial branching pattern to be unique justifying documentation in surgical literature.

The authors carried out a review of literature and found that the common arterial anomalies reported were persistent superficial brachial artery, high division of brachial, radial and ulnar arteries (2).

Variant arterial branching pattern of the upper extremity is often seen to co-exist with neural variations suggesting that the

Department of Anatomy, Vardhaman Mahavir Medical College, New Delhi, India

Address for correspondence: V. Mehta, Dr, MS, VMMC \& Safdarjung Hospital, New Delhi, India.

Phone: +9910061399 segmental origin of AA determines the branching pattern of brachial plexus during embryogenesis (3).

Anomalies of the branching pattern of axillary artery are of valued interest to clinicians including radiologists, plastic surgeons, anesthetists and orthopedic surgeons.

Therefore anatomists never cease to report any unusual pattern of ramification of this vital vessel of upper extremity with the belief that even the rarest of anomalies should be listed in anatomical records.

\section{Case report}

An instructive dissection of the right axilla revealed an uncharacteristic branching pattern of the second and third parts of axillary artery (AA). The first part of axillary artery was seen to extend as usual from the outer border of the first rib to the upper border of the pectoralis minor muscle (PMI). It gave off two branches : the superior thoracic (ST) and the thoraco-acromial (TA), the latter being normally a branch of the second part of AA (Fig. 1) The ST arose from the anterior aspect whereas the TA from the superior aspect of AA. The ST was seen to divide into transverse and descending branches on the anterior thoracic wall. Distally the AA divided into two trunks -medial and lateral, $4 \mathrm{~mm}$ proximal to the upper border of PMI (I \& II in Fig. 1). The lateral trunk continued into the arm as the main AA which laterally continued as the brachial artery at the lower border of teres major muscle. It was interposed 


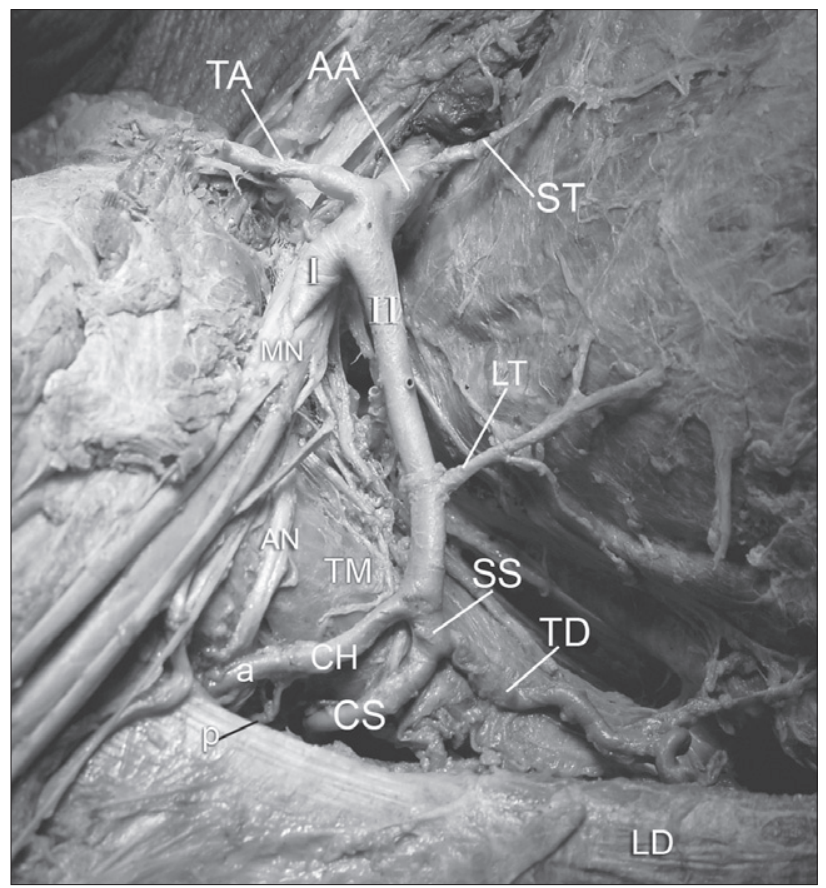

Fig. 1. Photograph of the right axillary region showing the following: AA - axillary artery, ST - superior thoracic artery, TA - thoraco acromial artery, LT - lateral thoracic artery, I- lateral trunk of second part of axillary artery, II - medial trunk of axillary artery, $\mathrm{CH}$ - circumflex humeral trunk, a - anterior circumflex humeral artery, $\mathbf{p}$ - posterior circumflex humeral artery, $\mathrm{SS}$ - subscapular artery, CS - circumflex scapular artery, $\mathrm{AN}$ - axillary nerve, $\mathrm{MN}$ - median nerve, $\mathrm{TM}$ - teres major muscle, $\mathrm{LD}$ - latissimus dorsi.

between the two roots of median nerve. The wider medial trunk ran deep to the PMI where it gave off the lateral thoracic artery and a muscular branch. The length of this medial trunk proximal to the origin of LTA was $4.5 \mathrm{~cm}$. Distaly the medial trunk gave off three branches distal to the PMI- anterior and posterior circumflex humeral and subscapular arteries (SS). However, the medial trunk continued for $42 \mathrm{~mm}$ (Fig. 1) before giving off the anterior and posterior circumflex humeral and subscapular arteries. The SS divided into throraco-dorsal and circumflex scapular arteries. The anterior circumflex humeral artery was thicker in caliber than the posterior circumflex humeral artery. The length of SS was $11 \mathrm{~mm}$. The course, distribution and branching pattern of the left AA was normal. No abnormal neural communications or an anomalous branch of the brachial plexus was observed. The axillary vein displayed usual anatomy in terms of course and tributaries.

\section{Discussion}

Deviations from the normal branching pattern of axillary artery are well reported in anatomical literature. A thorough knowledge of the possible arterial branching variations of the upper extremity is of immense relevance during reparative surgery in this region (4). Additionally, a variant arterial pattern of the upper extremity may cause complications while raising radial and ulnar artery flaps during plastic surgical procedures (5). Inadvertent usage of diagnostic and surgical techniques may not only render surgery difficult but may also lead to impediments such as hemorrhage from the variant vessels.

An embryological basis can be assigned to such arterial anomalies which may be an abnormal rate of growth and development from the seventh intersegmental artery or failure of absorption during formation of the limbs (6). Interestingly, there may also be an arrest at any stage of embryogenesis showing retention, reappearance or regression which may also amount to anomalous branching of the artery.

Studies in the past have reported high origin of radial and brachial artery along with bifurcation of AA into superficial brachial and a deep brachio-thoracic arteries as the common arterial variations of the upper extremity $(7,8)$. In one study the deep brachial artery which was the lateral stem gave off the circumflex and subscapular arteries. The other medial stem which was also superficial continued as the brachial artery (2). A similar bifurcation of AA into two equal sized trunks was observed in an earlier report.

However, they were disposed in superficial and deep planes. The superficial continued as the brachial artery and the abnormal deep trunk bifurcated into a common circumflex humeral- subscapular trunk. In our study specimen, the lateral trunk continued into the brachium and therefore may be regarded as a high origin of brachial artery. On the other hand the other trunk of equal caliber served as the regular AA, giving off branches which the second and third parts of AA normally give (9). A previous study reported an incidence of $11-24 \%$ for the arterial variations related to the upper limb. The present anomaly of duplication of second part of axillary artery however, is less commonly reported (10).

The fact that this particular anomaly has been noticed in only one cadaver makes it a rare and noteworthy variation demanding a place in anatomical archives and text books. In the present study, the second part of AA is dividing into the two trunks - AA I and AAII. We aimed to designate the AAI as AA proper and AAII as axillothoracic artery. The wide caliber of the second part of AAjust before bifurcation is also worth mentioning. It may be presumed that due to the anomalous branching pattern of the AA, its wide caliber serves as compensation in order that the vascular supply to the limb is not jeopardized. Furthermore, we cogitate that the AA which continues into the arm may not be of sufficient size and caliber to provide adequate vascular leverage to the extremity should there be any occlusion of the other trunk of axillary artery giving off the branches.

Vascular surgery entails two procedures namely Doppler pressure studies and Duplex ultrasound (11). Peripheral vascular injuries are ascertained by Duplex ultrasound whilst Doppler pressure studies are used for arterial pressure estimation. The interpretation of these angiographic studies is disturbed by the presence of such arterial anomalies (8). A pre-operative angiographic maneuver is mandatory in order to note such variants so that procedures such as cannulation and repairs are easier and hassle free. Additionally, the knowledge of the present variation is worth considering while performing several procedures such as anterograde cerebral perfusion in aortic surgery, axillary reconstructions and treatment of aneurysm of AA and radical mastectomy to list a few (3).

We also speculate that the peripheral arterial pulsation of upper limb would presumably be weak and could cause difficulty 
in blood pressure monitoring. Awareness of the altered vascular pattern was encountered in the present case and is of tremendous significance to vascular surgeons as well as to radiologists while interpreting angiographic studies of upper limb.

Knowing the possible variants of the AA branches and their relations to the brachial plexus is of value in clinical practice. Another comparable report noted the division of AA into regular and variant branches at the upper border of PMI (12). Nevertheless, we feel the arterial variations should be mentioned for the benefit of radiologists and operating surgeons to prevent any untoward complication resulting from lack of clinical anatomical knowledge.

\section{Conclusion}

Knowledge about variations in the branching patterns of axillary artery has an important bearing on the interpretation of radiological studies and surgical outcome. We suggest documentation of any arterial anomaly for precise clinical diagnosis and surgical interventions.

\section{References}

1. Williams PL, Bennister LH, Berry MM, Collins R, Dyson M, Dussek JE, Ferguson MW. J. Gray's Anatomy. In: Cardiovascular system. Gabella G (Ed). 38th Ed. Edinburg: Churchill Livingstone, 1995, 1537-1538.

2. Cavdar S, Zeybek A, Bayramicli M. Rare variation of the axillary artery. Clin Anat 2000; 13: 66-68.
3. Bhat KMR, Gowda S, Potu BR, Rao MS. A unique branching pattern of the axillary artery in a south indian male cadaver. Bratisl Lek Listy 2008; 109 (12): 587-589.

4. Mehta V, Arora J, Suri RK, Rath G. Unialteral anomalous arterial pattern of human upper limb: anatomical description and clinical implications. Sultan Qaboos Med J 2008; 8 (2): 95-98.

5. Fatah MF. Raising the radial artery forearm flap: superficial ulnar artery “trap". Br J Plast Surg 1985; 38 (3): 394-395.

6. Tan CB, Tan CK. An unusual course and relations of human axillary artery. Sing Med J 1994; 35: 263-264.

7. Yotova N, Novakov S, Upper limb double axillary artery, Clin Anat 2004; 17: 149-151.

8. Compta XG. Origin of radial artery from axillary artery and associated hand abnormalities. J Hand Surg (Am) 1991; 16 A: 293-296.

9. George BM, Nayak S, Kumar P. Clinically significant neurovascular variations in the axilla and the arm. Neuroanatomy 2007; 6 (1): 36 .

10. Uglietta JP, Kadii S. Arteriographic study of variant arterial anatomy of upper extremities; cardiovascular intervention. Radiology1986; 12: $145-148$.

11. Jayakumari S, Rath G, Arora J. Upper limb double axillary artery and double brachial arteries, embryological basis and its clinical implications, Int J Morphol 2006; 24 (3): 463-468.

12. Yotova N, Novakov S. Unilateral double axillary arteries. Clin Anat 2004; 17: 149-151. 